\title{
Health network mergers and hospital re-planning
}

\author{
ED Güneş ${ }^{1 *}$ and $\mathrm{H}$ Yaman $^{2}$ \\ ${ }^{1}$ Koc University, Istanbul, Turkey; and ${ }^{2}$ Bilkent University, Ankara, Turkey
}

This paper presents an integer programming formulation for the hospital re-planning problem which arises after hospital network mergers. The model finds the best re-allocation of resources among hospitals, the assignment of patients to hospitals and the service portfolio to minimize the system costs subject to quality and capacity constraints. An application in the Turkish hospital networks case is illustrated to show the implications of consolidation of health insurance funds on resource allocations and flow of patients in the system.

Journal of the Operational Research Society (2010) 61, 275-283. doi:10.1057/jors.2008.165

Published online 11 February 2009

Keywords: hospital networks; merger and acquisition; hospital planning; quality constraints; resource allocation

\section{Introduction}

Merger and acquisition (M\&A) activity has been increasing in recent years in many industries including health services (Japsen, 1996; The Economist, 1999). Mergers are expected to bring efficiency gains in service or product delivery systems. However, most of M\&As seem to deliver disappointing outcomes if they are to be evaluated on the basis of efficiency or profitability as reported by Weil (2000).

Failures of mergers can be attributed to many reasons. The lack of coordination and centralized control may be one obstacle to fully benefit from mergers (Clement et al, 1997). Although there can be managerial and cultural reasons to this problem, in this paper we approach this issue from an operational perspective. The question we are interested in is, what is the potential benefit of full coordination and centralized control, and how should it be realized. We interpret 'coordination' as re-allocating resources and patients within the network, and model this problem using an optimization approach.

Our motivation comes from the health system transformation project in Turkey. One component of the project was defined as integration and harmonization of Ministry of Health (MoH) and Social Security Institute (SSK) hospitals towards greater autonomy' (World Bank, 2004). When this component was implemented in 2005, all the SSK hospitals were acquired by the $\mathrm{MoH}$, thus effectively merging the two hospital networks. Before this merger, SSK and MoH hospitals served separate populations. After the merger, members of both populations have the right to get service from any hospital within the merged network. This means that previously unrelated hospitals are now linked, creating an

*Correspondence: ED Güneş, College of Administrative Sciences and Economics, Koc University, Rumeli Feneri Yolu, 34450 Sariyer, Istanbul, Turkey.

E-mail: egunes@ku.edu.tr opportunity to re-design the system. Moreover, the new system introduces the referral of patients to hospitals by family physicians. This makes it possible to impose an assignment of patients to specific hospitals to optimize the system.

The existing health system faced many problems including the ineffective geographical distribution of resources (Morlock et al, 2004), and the under-utilization of hospitals because of their small size and lack of resources in rural areas (World Bank, 2003). There is also an ineffective allocation of resources: A hospital may have a general surgeon but not an anaesthesiologist, which makes it impossible to perform operations. On the other hand, after the merger there is a possibility of unnecessary duplication of resources; this should be avoided (Morlock et al, 2004). Existing doctors and hospital beds can be re-allocated within the merged network to tackle these problems. Since the doctors in these hospitals work for the government, their posts can be changed if needed.

We present a hospital re-planning problem that could be encountered in any country where hospital networks are merged, and study efficiency gains obtained by M\&As in hospital networks. To this end, we provide an integer programming model for the problem, and apply this model using data from Turkish hospitals.

Our model takes into account several features related to health systems: (1) enough demand is assigned to a hospital service so that physicians can maintain service quality, (2) availability of all resources required for a service is ensured and (3) possibility to investigate whether to close hospitals is provided. The aim of our model is to minimize the total system cost, that is, the sum of the cost of changes in the hospital network and the cost of access to service for the patients.

The model is also applicable to mergers of supply chain systems. It may be used to re-allocate resources and assign demand to facilities to minimize the total distribution cost of the merged supply chain. 
The model focuses on cost minimization instead of benefit maximization. One cost component is patients' cost of access to service. Minimizing this is equivalent to maximizing benefits for patients since we assume that service quality is the same at all hospitals.

The rest of the paper is organized as follows: Section 2 reviews the literature. Section 3 introduces the model formulation. In Section 4, we apply the model using hospital data from a region of Turkey and show how much potential improvement can be gained by consolidation of Turkish hospital networks. We conclude in Section 5.

\section{Literature review}

There are two streams of research related to our problem. One is concerned with performance effects of M\&As and mainly uses empirical methods; the other focuses on hospital planning problems and uses facility location-allocation models.

M\&A activity has been analysed from various perspectives in the general management literature. Some have studied the benefits to shareholders from acquisitions, and found that most of the gains accrue to the targets (Gupta and Gerchak, 2002). Connor et al (1997) analysed M\&As in health networks and showed that higher merger-related savings are accomplished by lower occupancy hospitals. The drivers of success in hospital mergers are discussed by Weil (2000); utilization patterns, financial ratios, corporate culture and contracts are mentioned as important factors.

Harris et al (2000) investigated the gains in technical efficiency after hospital mergers using longitudinal data envelopment analysis (DEA). Sherman and Rupert (2005) used DEA to analyse a 200-branch network formed after a merger of four banks, and found opportunities to reduce operating costs after the merger. Few papers have modelled mergers in an analytical framework, and they considered a production system as opposed to a health system (Gupta and Gerchak, 2002; Iyer and Jain, 2004).

Location-allocation models have been used quite extensively for quantitative analysis in health services (Rahman and Smith, 2000). A common objective is minimizing travel cost (Rahman and Smith, 1999; Chu and Chu, 2000; Galvao et al, 2002). A multi-objective heuristic approach for the location-allocation decisions in a hospital network is introduced by Stummer et al (2004). Dokmeci (1977) introduces a quantitative model to decide facility location and capacities in a hierarchical health care system.

Bretthauer and Cote (1998) provide an extensive review of capacity management in health services. Few papers have focused on the resource re-allocation in existing facilities. Ruth (1981) models re-allocation of only beds using a mixedinteger programming approach within an existing hospital network. Geller and Yochmowitz (1975) looked at reduction of maternity beds in a region and investigated bed closing rules.
Verter and Lapierre (2002) model the problem of locating preventive health care facilities with the objective of maximizing participation, with the requirement of assigning more than a certain number of individuals to a facility because of quality concerns. Harper et al (2005) model a stochastic hospital planning problem with a discrete-event simulation approach. Güneş and Yaman (2005) study a problem similar to the one presented here, propose a model and present preliminary results for the case where only beds can be re-allocated.

Here, we give a model which considers the impacts of closing hospitals, incorporates bounds on number of resources that can be transferred, and proposes a more realistic view of modelling capacities by considering different types of facilities as space, number of rooms, etc. We present extensive numerical results for the setting where two types of resources, beds and specialist doctors can be re-allocated and show that considerable amounts of improvement can be achieved by reallocation of specialist doctors. We also perform sensitivity analysis to observe the quality/decentralization trade-off and the effect of cost parameters on the level of improvements achieved.

\section{Problem definition and formulation}

Our objective in this study is to find a better resource allocation after a merger of two networks and thus understand the potential cost benefits of the merger. To this end, we model the optimization of resource re-allocation in the merged hospital network. The parameters of the problem are given in Table 1.

\subsection{Assumptions on hospitals and resources}

Let $H$ denote the set of hospitals and $P$ the set of population centers. The hospitals offer a set of services, denoted by $S$, and these services require a set of resources, denoted by $R$. Each service $s \in S$ requires a certain set of resources, $R_{S} \subseteq R$, and these sets may not be mutually exclusive, that is, some resources can be used for more than one service. For a hospital network, the services are specialty services like radiology, brain surgery. Examples of the resources are specialist doctors, nurses, equipment and beds. For each service, certain resources are required; for example, for the mammogram service, a radiologist, a technician and a mammography machine are needed. A hospital bed can be used by any inpatient service, that is, a resource can be required for more than one service.

Each resource has a certain capacity to serve patients for a service; $\kappa_{s r}$ is the amount of resource $r \in R_{s}$ required to serve a patient in service $s \in S$. Each hospital has facilities to hold different types of resources. We denote by $C$ the set of facility types (like space, number of rooms or offices, etc), by $K_{i c}$ the capacity of facility type $c \in C$ at hospital $i \in H$, by $\prod_{c} \subseteq R$ the set of resources that use facility type $c \in C$ and by $\omega_{r c}$ the amount of facility of type $c \in C$ that a unit resource $r \in R$ uses. 
Table 1 Problem parameters

$P \quad$ the set of population centres

$H \quad$ the set of hospitals

$R \quad$ the set of resources

$S \quad$ the set of services

$C \quad$ the set of facilities used to accommodate resources in hospitals

$H_{i} \quad$ the set of hospitals to which resources can be transferred from hospital $i \in H$

$R_{S} \quad$ the set of resources that are required for service $s \subseteq S$

$C_{r i} \quad$ the amount of resource $r \in R$ available at hospital $i \in H$ before the re-planning

$\kappa_{s r} \quad$ the amount of resource $r \in R_{S}$ required to serve a patient in service $s \in S$

$\prod_{c} \quad$ the set of resources that use facility type $c \in C$

$\omega_{r c} \quad$ the amount of facility type $c \in C$ that a unit resource $r \in R$ uses

$D_{k s} \quad$ the number of patients in population centre $k \in P$ who need service $s \in S$

$K_{i c} \quad$ the capacity of facility type $c \in C$ at hospital $i \in H$

$t_{k i} \quad$ the cost of travelling from population centre $k \in P$ to hospital $i \in H$

$a_{\text {rij }} \quad$ the cost of transferring a unit resource $r \in R$ from hospital $i \in H$ to hospital $j \in H_{i}$

$p_{i} \quad$ the cost of closing hospital $i \in H$

$m_{1 s} \quad$ the minimum number of patients that should be served by service $s \in S$ in any hospital

$m_{2}$ the minimum number of services to be offered in an open hospital

$\rho_{s i} \quad$ the maximum acceptable ratio of demand to capacity for service $s \in S$ at hospital $i \in H$

$\alpha_{r i} \quad$ the maximum fraction of resource $r \in R$ that can be transferred from hospital $i \in H$

$k_{r i j} \quad$ the maximum amount of resource $r \in R$ that can be transferred from hospital $i \in H$ to hospital $j \in H_{i}$

$C_{r i}$ denotes the amount of resource $r \in R$ available at hospital $i \in H$ before the re-planning. The resources at hospital $i \in H$ that can be transferred to a set of hospitals are denoted by $H_{i} \subseteq H$. The cost of transferring a unit resource $r \in R$ from hospital $i \in H$ to hospital $j \in H_{i}$ is $a_{r i j}$. The amount of resource $r \in R$ transferred from a particular hospital $i \in H$ cannot exceed a certain fraction, $\alpha_{r i}$ of the existing resource at hospital $i$ if the hospital remains open. This is a limitation that may be imposed to minimize detraction from resource transfers in the hospitals. There may also be an upper bound on the amount of resource $r$ transferred from hospital $i \in H$ to hospital $j \in H_{i}$, denoted by $k_{r i j}$. Such a constraint may minimize the problem of resistance from doctors who do not want to be transferred to certain rural locations by not transferring too many doctors from the same hospital.

\subsection{Assumptions on assignment of patients to hospitals}

Each population centre generates a certain demand for each hospital service. We assume a utilization target is set in order to cope with the variabilities in the system, that is, there is a maximum acceptable ratio of demand to capacity for service $s \in S$ at hospital $i \in H$ denoted by $\rho_{s i}$. This is an approach used by others (Stummer et al, 2004) to model similar problems in deterministic settings. The demand of population centre $k \in P$ for service $s \in S$ is denoted by $D_{k s}$ and the cost of transportation for a patient between population centre $k \in P$ and hospital $i \in H$ is denoted by $t_{k i}$. This cost is typically a linear function of the distance or travel time. If cover type constraints are imposed, then it is possible to change this cost accordingly. For instance, if every patient should be able to get service within $M$ kilometers from his/her population centre, then $t_{k i}$ can be a linear function of distance for distances less than or equal to $M$ but it may be infinity for larger distances. It is also possible to use more sophisticated transportation cost functions, such as adding a fixed cost for travelling in addition to a variable transportation cost which may be a piecewise linear function of the distance. The transportation costs between two population centres are considered as parameters in our model, and the shape of the function does not affect the model.

For hospital services, a minimum number of patients, defined by a standard, should be served each year so that a good quality of service can be maintained. Therefore, a service can be offered at a hospital only if a minimum demand is assigned to this hospital. This constraint can be tight for some services, like screening mammogram, or surgery, where physicians learn from practice. For other services like emergency care, such a constraint may not be applicable at all. This type of constraint was also used by Verter and Lapierre (2002) in their model of location of mammogram centres. Another reason to impose such a constraint can be to justify the fixed costs of offering that service. We denote this minimum value by $m_{1 s}$ for service $s \in S$.

We assume that for a hospital to remain open after replanning, at least $m_{2}$ services should be offered in this hospital, otherwise the cost of closing the hospital $i \in H$ is $p_{i}$. This constraint makes the model useful for general hospitals, which should offer a variety of services. If such a constraint does not apply, it can be relaxed by setting $m_{2}=1$.

\subsection{Decision variables and the objective function}

The decisions centre around the re-allocation of resources to hospitals, the set of hospitals that remain open and their service portfolios and the assignment of patients to hospitals for different services. We use the following decision variables 
in our model. We define $x_{r i j}$ as the amount of resource $r \in R$ transferred from hospital $i \in H$ to hospital $j \in H_{i}, z_{k s i}$ as the demand from population centre $k \in P$ assigned to hospital $i \in H$ for service $s \in S$, and $E_{s i}$ as the maximum number of patients that can be treated at service $s \in S$ at hospital $i \in H$ after the re-planning. The binary variable $u_{s i}$ is 1 if service $s \in S$ is offered at hospital $i \in H$ and 0 otherwise and $y_{i}$ is 1 if hospital $i \in H$ remains open and 0 otherwise.

The objective is to minimize the total cost which is composed of the cost of transferring resources, the cost of closing hospitals and the cost of transportation of patients. In fact, these costs are incurred by different parties. Typically, the system design costs (resource transfers and hospital closures) are incurred by the decision maker (the state, or the management of the health network), while the patient transfer costs are incurred by the patients. Since our objective is to optimize system-side performance, all cost components are included in the objective function. As a policy maker, the weight of patient transportation cost in decision making can be changed giving different weights to the unit cost figures. If the state has a budget for the project of re-designing the health network, then the model can be modified easily to minimize the patients travel costs subject to a budget constraint on the design costs. Alternatively, if the decision maker is interested in minimizing system design costs subject to cover type constraints, this can be handled by assigning values to the parameters $t_{k i}$ 's as explained in Subsection 3.2. The hospital re-planning problem can be modelled as follows:

$$
\begin{array}{ll}
\min & \sum_{r \in R} \sum_{i \in H} \sum_{j \in H_{i}} a_{r i j} x_{r i j}+\sum_{i \in H} p_{i}\left(1-y_{i}\right) \\
& +\sum_{k \in P} \sum_{i \in H} t_{k i} \sum_{s \in S} z_{k s i} \\
\text { s.t. } & \sum_{j \in H_{i}} x_{r i j} \leqslant \alpha_{r i} C_{r i} y_{i}+C_{r i}\left(1-y_{i}\right) \quad \forall r \in R, i \in H \\
& \sum_{i \in H} z_{k s i}=D_{k s} \quad \forall k \in P, s \in S \\
& \sum_{s \in S: r \in R_{s}} \kappa_{s r} E_{s i} \leqslant C_{r i}+\sum_{j \in H: i \in H_{j}} x_{r j i}-\sum_{j \in H_{i}} x_{r i j} \\
& \forall r \in R, i \in H \\
& \sum_{k \in P} z_{k s i} \leqslant \rho_{s i} E_{s i} \quad \forall s \in S, i \in H \\
& \sum_{r \in \prod_{c}} \omega_{r c}\left(C_{r i}+\sum_{j \in H: i \in H_{j}} x_{r j i}-\sum_{j \in H_{i}} x_{r i j}\right) \leqslant K_{i c} \\
& \forall i \in H, c \in C \\
& \sum_{k \in P} z_{k s i} \geqslant m_{1 s} u_{s i} \quad \forall s \in S, i \in H
\end{array}
$$

$$
\begin{aligned}
& z_{k s i} \leqslant D_{k s} u_{s i} \quad \forall k \in P, s \in S, i \in H \\
& \sum_{s \in S} u_{s i} \geqslant m_{2} y_{i} \quad \forall i \in H \\
& u_{s i} \leqslant y_{i} \quad \forall s \in S, i \in H \\
& x_{r i j} \leqslant k_{r i j} \quad \forall r \in R, i \in H, j \in H_{i} \\
& u_{s i} \in\{0,1\} \quad \forall s \in S, i \in H \\
& y_{i} \in\{0,1\} \quad \forall i \in H \\
& x_{r i j} \in \mathbb{Z}_{+} \quad \forall r \in R, i \in H, j \in H_{i} \\
& z_{k s i} \in \mathbb{Z}_{+} \quad \forall k \in P, s \in S, i \in H
\end{aligned}
$$

Constraints (2) ensure that the amount of resources transferred from a hospital cannot exceed a fraction of the initial amount of that resource at that hospital if the hospital remains open. Constraints (3) imply that all demand is served. Owing to constraints (4), the sum of the final capacities of services using a resource cannot exceed the final amount of that resource. The final amount of a resource is the previous amount plus the net amount of transfers. Constraints (5) state that the existing capacity multiplied with a maximum utilization factor $\rho_{s i}$ for a service should be greater than or equal to the demand assigned to that service. Constraints (6) imply that the total capacity requirement of the resources using a given type of facility should not exceed the available capacity at that hospital. Owing to constraints (7), for service $s$ to be provided in a hospital, the hospital should serve a minimum demand of $m_{1 s}$ units. Constraints (8) ensure that if a service is not provided in a hospital, no demand is assigned to that hospital for that service. Constraints (9) imply that in each hospital that remains open, a minimum of $m_{2}$ services are offered. Owing to constraints (10), if a hospital is closed, no service is offered at this hospital. Constraints (11) ensure that no more than a predetermined maximum amount of resources are transferred between a pair of hospitals. Constraints (12)-(15) are integrality and $0-1$ requirements.

Our model has $\mathrm{O}(|H|(1+|S|+|R||H|+|P||S|))$ variables and $\mathrm{O}(|H|(1+|C|+2|R|+3|S|+|P||S|+|R||H|)+|P||S|)$ constraints.

The model does not forbid unused resources from remaining at hospitals which have closed. If all resources were to be transferred to hospitals that remain open after the re-planning, then constraints (6) should be changed with

$$
\begin{aligned}
& \sum_{r \in \prod_{c}} \omega_{r c}\left(C_{r i}+\sum_{j \in H: i \in H_{j}} x_{r j i}-\sum_{j \in H_{i}} x_{r i j}\right) \leqslant K_{i c} y_{i} \\
& \forall i \in H, c \in C
\end{aligned}
$$

These new constraints imply that for $i \in H$ whenever $y_{i}$ is zero, the final amount of resource $r$ at hospital $i$, that is, $C_{r i}+\sum_{j \in H: i \in H_{j}} x_{r j i}-\sum_{j \in H_{i}} x_{r i j}$ is zero for all $r \in \prod_{c}$ and $c \in C$. 
It is also possible to apply different rules for different types of resources. For instance, if a hospital is closed, then it may be necessary to re-allocate all specialist doctors, but beds that are not required may remain. We can model the requirement that all the amount of resource $r \in R$ needs to be re-allocated if hospital $i \in H$ is closed by adding the constraint $\sum_{j \in H_{i}} x_{r i j} \geqslant C_{r i}\left(1-y_{i}\right)$. If $y_{i}=0$, this constraint becomes $\sum_{j \in H_{i}} x_{r i j} \geqslant C_{r i}$. This together with constraint (2) implies $\sum_{j \in H_{i}} x_{r i j}=C_{r i}$ stating that all available resource $r$ should be transfered to other hospitals. If $y_{i}=1$, then the constraint is redundant.

If the wish is not to close any hospital, then constraints (9) are replaced with $\sum_{s \in S} u_{s i} \geqslant \max \left\{m_{2}, 1\right\}$ for all $i \in H$, variables $y_{i}$ are fixed to 1 , and constraints (10) are removed. Some services may be required to exist in any hospital that remains open. If service $s \in S$ is such a service, then constraint (10) for hospital $i \in H$ should be replaced with $u_{s i}=y_{i}$.

Note that there is an inherent tension between the desire not to close any hospitals and the need to have a high number of patients served in all hospitals to increase quality. This tradeoff is reflected in the parameters $m_{2}$ and $m_{1 s}$, respectively. A high $m_{1 s}$ implies more volume, hence better quality with increased centralization. However, a high $m_{2}$ pushes for more decentralized service to increase accessibility to services. The model parameters must be set by the policy makers, or the hospital network managers, reflecting their strategic choice given this trade-off. Finally, note that estimating parameters may be the most challenging part of implementing the model in practice. These weights represent strategic choices between quality and access, or costs to the hospitals and costs to the patients, which are political issues open to debate.

\section{Case study}

In this section we present an application of the model for a case of Turkish hospitals in 11 cities. The Turkish Health System is organized in regional health planning centres. These 11 cities are those that are governed by one of the planning centres and they are all located in the central-western Anatolia.

Our choice of services and resources to include in the study was limited by the availability of data. We focus on two basic resources: hospital beds and specialist doctors for each service. Five inpatient services are considered: (1) internal medicine, (2) general surgery, (3) ear, nose and throat, (4) orthopaedics and (5) urology. Since each specialist doctor can be used only for their respective service, we have six different resources in the analysis. Below, first we explain the data set used, and then discuss the results.

\subsection{Demand estimation}

The demand data are estimated using data given in the Statistics Yearbook (2004) published online by the Turkish Ministry of Health. We first estimated the total demand in each city, then allocated that to the two insurance funds, SSK and MoH. The procedure is as follows: Number of inpatient discharges
Table 2 Summary information on the two hospital networks

\begin{tabular}{|c|c|c|c|c|c|c|}
\hline & Bed & INM & $G S U$ & ENT & ORT & $U R O$ \\
\hline \multicolumn{7}{|c|}{ Capacity (number of resources) } \\
\hline SSK & 2145 & 83 & 106 & 64 & 66 & 60 \\
\hline $\mathrm{MoH}$ & 3676 & 154 & 237 & 143 & 120 & 117 \\
\hline \multicolumn{7}{|c|}{ Capacity (patients per year) } \\
\hline SSK & 156585 & 43824 & 38584 & 14400 & 25674 & 18840 \\
\hline $\mathrm{MoH}$ & 268348 & 81312 & 86268 & 32175 & 46680 & 36738 \\
\hline \multicolumn{7}{|c|}{ Demand (patients per year) } \\
\hline SSK & 129537 & 43606 & 41044 & 12824 & 19239 & 12824 \\
\hline $\mathrm{MoH}$ & 229796 & 78674 & 72657 & 21499 & 35467 & 21499 \\
\hline
\end{tabular}

The specialist doctors are labelled as INM: internal medicine, GSU: general surgery, ENT: ear, nose and throat, ORT: orthopaedics, URO: urology.

and deaths for each city and percentage of patients for each service among all inpatient demand are given in the yearbook. Multiplying total demand with percentage figures, we found the total number of patients for each service in each city. The number of employees insured by the SSK fund in a given city is reported by the Social Security Institute. We multiplied that with $4^{1}$ to reflect the family of those employees who benefit from the fund. The ratio of this number to the population size gave us the percentage of patients that are insured by SSK in each city. All the remaining patients use the MoH hospitals. We should note that using the historical data on number of inpatient discharges underestimates the demand, since the demand that could not be met is actually lost, but this is the only data available to us. A summary of the general characteristics of the two networks are given in Table 2 .

\subsection{Capacity estimation}

We obtained the data on capacities of the hospitals in the region from the Turkish Ministry of Health. Among those hospitals only the general hospitals are considered in the analysis. There are 16 hospitals in the SSK network and 31 hospitals in the MoH network. Together they comprise 5821 hospital beds and 1150 specialist doctors. The raw data were given in terms of the number of beds and the number of specialist doctors; we converted them to units of "number of patients per year'. Average length of stay was given as 5 days in the Statistics Yearbook (2004), so capacity of a bed is set to $365 / 5=73$ patients/year. The number of beds that a specialist can serve is set to the average number of beds per specialists for each department, calculated using the total number of beds and specialists data. Then that number is multiplied by 73 to find the number of patients per doctor for each specialist group. Table 2 gives the capacity figures used. Table 3 gives the number of beds and the number of specialist doctors per 100000 people for each city in consideration, which

\footnotetext{
1 The number of dependents per insured for the year 2004 was found to be 4.4 according to the statistics provided by SSK in 2007 (SSK, 2007); hence 4 is a reasonable approximation.
} 
Table 3 Summary information on distribution of resources among cities

\begin{tabular}{lrlllll}
\hline City & Bed & INM & GSU & ENT & ORT & URO \\
\hline Number of resources per & 100000 people & & & \\
Ankara & 62.38 & 2.87 & 5.19 & 3.22 & 2.79 & 2.74 \\
Afyon & 58.22 & 1.97 & 1.97 & 1.11 & 1.11 & 0.98 \\
Corum & 66.99 & 1.84 & 2.34 & 1.34 & 1.00 & 1.34 \\
Eskisehir & 62.46 & 2.83 & 2.55 & 1.56 & 1.42 & 1.42 \\
Kastamonu & 38.88 & 1.60 & 2.40 & 1.07 & 1.60 & 0.80 \\
Kutahya & 75.81 & 2.74 & 2.59 & 1.22 & 1.67 & 1.07 \\
Zonguldak & 89.67 & 3.41 & 3.25 & 1.95 & 1.79 & 1.62 \\
Bartin & 85.79 & 7.60 & 8.69 & 6.52 & 5.43 & 4.34 \\
Kirikkale & 70.66 & 1.56 & 2.61 & 1.56 & 0.78 & 1.56 \\
Karabuk & 111.95 & 3.55 & 5.78 & 2.67 & 2.22 & 2.22 \\
Cankiri & 48.08 & 0.74 & 0.74 & 0.74 & 1.11 & 0.74 \\
\hline
\end{tabular}

The specialist doctors are labelled as INM: internal medicine, GSU: general surgery, ENT: ear, nose and throat, ORT: orthopaedics, URO: urology.

demonstrates the uneven distribution of the existing capacity in different cities. The demand and capacity data are available online at http://www.bilkent.edu.tr/ hyaman/health.htm.

\subsection{Parameter estimation}

We fixed the following parameters. We use $\rho_{s i}=1.1$ for all $s \in S$ and $i \in H$ as there is a capacity shortage in the SSK network for one of the resources (see Table 2). This is not so unrealistic as the problem of overloaded doctors in the SSK hospitals has been a well-known issue. The resource transfer cost for beds and transportation costs are functions of the travel time. Let $d_{i j}$ denote the travel time between hospital $i \in H$ and $j \in H$ and $d_{k i}^{\prime}$ denote the travel time between population centre $k \in P$ and hospital $i \in H$. We take the cost of transferring a bed from hospital $i$ to hospital $j$ as $a_{1 i j}=0.1 d_{i j}$ and the travelling cost from population centre $k$ to hospital $i$ as $t_{k i}=d_{k i}^{\prime}$. As will be discussed in the application results, conclusions do not seem to be sensitive to these parameters. The transfer cost for doctors does not depend on the distance and is taken to be 1000. This is the value paid to a doctor whose post is changed in the Turkish case. We experimented with fixing the value of $m_{1 s}=100$ for all $s \in S$ and changing the value of $m_{2}$ to $1,2,3$ and 4. The cost values did not change significantly. For $m_{2}=4$, no feasible patient assignment could be found for the SSK network and the merged network if resource transfers were forbidden. In the remaining, we take $m_{2}=1$. Since we would like all hospitals to remain open, we modify the formulation accordingly as explained in Section 3. As we are interested in the potential cost improvement, we assumed there is no constraint on the maximum number of resources that could be transferred, and interpret the result as an upper-bound on the cost reduction. We also assumed that the doctors would accept to move to their new posts, which is reasonable since doctors work for the government. Therefore we set $\alpha_{r i}=1$ and $k_{r i j}=C_{r i}$ for all $r \in R, i \in H$ and $j \in H_{i}$ and remove the redundant constraints.
Table 4 Effect of resource transfers in the three networks

\begin{tabular}{|c|c|c|c|c|c|c|}
\hline \multirow[t]{2}{*}{ Network } & \multirow[t]{2}{*}{$m_{1 s}$} & \multicolumn{3}{|c|}{$\%$ Improvement in } & \multicolumn{2}{|c|}{ \# Transfers of } \\
\hline & & $\begin{array}{c}\text { Total } \\
\text { cost }\end{array}$ & $\begin{array}{c}\text { Patient } \\
\text { travel } \\
\text { cost }\end{array}$ & $\begin{array}{l}\text { \# Patients } \\
\text { travelling }\end{array}$ & Beds & Doctors \\
\hline \multirow{3}{*}{$\mathrm{MoH}$} & 100 & 70.98 & 72.05 & 63.28 & 88 & 85 \\
\hline & 200 & 70.89 & 71.91 & 63.29 & 86 & 81 \\
\hline & 400 & 73.01 & 73.97 & 66.17 & 86 & 82 \\
\hline \multirow{3}{*}{ SSK } & 100 & 57.76 & 58.71 & 37.39 & 186 & 50 \\
\hline & 200 & 57.83 & 58.80 & 37.47 & 116 & 53 \\
\hline & 400 & 58.30 & 59.07 & 38.51 & 107 & 42 \\
\hline \multirow{3}{*}{ Merged } & 100 & 78.51 & 80.11 & 67.01 & 40 & 48 \\
\hline & 200 & 78.41 & 80.11 & 67 & 39 & 51 \\
\hline & 400 & 80.69 & 82.51 & 70.26 & 38 & 65 \\
\hline
\end{tabular}

The analysis involves a comparison of three problem instances, which correspond to three different hospital networks; 'SSK', 'MoH' and 'Merged' refer to the hospital network of the Social Security Institute, the network of the Ministry of Health hospitals and the merged network, respectively. We focus on two main mechanisms that reduce network costs. First, by transferring resources between hospitals, the network can be re-designed. This can be done within any given network, without the need of a merger. Second, by combining two hospital networks, transportation costs can be reduced by a better assignment of demands to hospitals. We refer to these two mechanisms as 'Resource Transfer' and 'Merging', respectively. In the following, we discuss the effect of these on the numerical analysis.

The integer programs are solved using CPLEX 9.0.2 with an optimality gap tolerance of $1 \%$. The largest model (Merged) consists of around 16000 variables and 4000 constraints, and the longest computation time is $1.73 \mathrm{~h}$.

\subsection{Effect of resource transfer}

Resource transfers between hospitals imply a re-allocation in the network, given the locations and capacity limitations at each location. In this section, we investigate the cost reduction from transferring hospital beds and specialist doctors between hospitals.

4.4.1. Effect on total cost Table 4 shows the percentage improvements in total cost, patient travel cost and the number of patients travelling to cities other than their population centres to get service. It also shows the number of beds and specialist doctors transferred to achieve these improvements. Thus the cost reduction achieved by resource transfers is about $70 \%$ for the Ministry of Health network and $57 \%$ for the Social Security Institute network. After merger, the benefits from resource transfer increase to $80 \%$. The improvements from transferring the given number of beds and doctors for each case are calculated with respect to the best patient assignment costs without resource transfers. 
The merged network gets the most benefit from resource transfers; the SSK network gets the least. This can be explained by the different sizes of these three networks: as the number of hospitals in the network increases, there are more alternatives for transfers, hence more opportunity for improvement. Also, the $\mathrm{MoH}$ network has lower utilization levels for specialist doctors, allowing for more flexibility in moving resources.

The number of transfers made and the cost reduction achieved are not correlated; the merged network can achieve the highest improvement by making the least number of resource transfers. Finally, we observe that improvement in patient travel cost is more than the improvement in the number of patients travelling to another population centre. This shows that by transferring resources, there is a decrease not only in the number of patients travelling to another city but also in the average distance travelled by these patients.

The minimum number of patients to be assigned to a service, $m_{1 s}$, does not have a significant impact on the effect of resource transfer when the number is 100-400. There is only a slight gain in cost reduction with a higher $m_{1 s}$.

Table 5 Effect of transferring only specialist doctors

\begin{tabular}{lccc}
\hline \multirow{2}{*}{ Network } & \multicolumn{3}{c}{ \% Improvement in } \\
\cline { 2 - 4 } & Total cost & Patient travel cost & \# Patients travelling \\
\hline MoH & 61.69 & 62.65 & 46.55 \\
SSK & 35.21 & 35.94 & 15.70 \\
Merged & 71.17 & 72.60 & 53.96 \\
\hline
\end{tabular}

Table 6 Effect of transferring only beds

\begin{tabular}{lccc}
\hline Network & \multicolumn{3}{c}{ \% Improvement in } \\
\cline { 2 - 4 } & Total cost & Patient travel cost & \# Patients travelling \\
\hline MoH & 0.28 & 0.28 & 0.55 \\
SSK & 7.78 & 7.78 & 6.61 \\
Merged & 0.00 & 0.00 & 0.00 \\
\hline
\end{tabular}

Next, we investigate the benefits when we transfer only one type of resources, either beds or specialist doctors. Here we take $m_{1 s}=200$ for all $s \in S$. Tables 5 and 6 summarize the results; most of the resource transfer benefits are generated by transferring doctors, while bed re-allocation brings a slight benefit. This is reasonable as beds are shared by the five services in consideration whereas doctors serve only their specialty services. This may act in combination with a good initial distribution which does not give much leeway for improvement.

4.4.2. The quality/decentralization trade-off The quality/ decentralization trade-off can be studied by comparing results of instances with different $m_{1 s}$ and $m_{2}$ values. Small $m_{1 s}$ values imply less strict quality constraints; $m_{2}=0$ implies hospital closures are allowed. We compared cases in which hospital closures were not allowed $\left(m_{2}>0\right)$ and $m_{1 s}$ value was small $(100,200,400)$ with cases in which $m_{2}=0$ and $m_{1 s}$ value was large (1000). In the latter case, a feasible solution can be achieved only after resource transfers. But as expected, when $m_{1 s}$ is large, the total cost of the merged network is higher than the cost of the network when $m_{1 s}$ is smaller. Increasing $m_{1 s}$ from 400 to 1000 increases total costs by about $22 \%$.

4.4.3. Sensitivity to unit resource transfer costs The last experiment in this section investigates the effect of resource transfer costs. We multiply the unit resource transfer costs by a coefficient which takes values of 0,1 and 10 . Table 7 shows that the improvement in patient travel cost is quite close in all cases. There are significantly more transfers when the resource transfer cost is 0 , since it represents the extreme case of free resource movements. However, a 10-fold increase in the resource transfer cost (from 1 to 10) does not have as much effect on the number of transfers made. This suggests that these transfers are quite useful in reducing the total system costs and the solution is hardly sensitive to the estimation of these costs.

Finally, we note that no feasible patient assignment exists in these networks for $\rho_{s i}=1$ for $s \in S$. However after resource

Table 7 Effect of resource transfer cost coefficients

\begin{tabular}{|c|c|c|c|c|c|c|}
\hline \multirow[t]{2}{*}{ Network } & \multirow{2}{*}{$\begin{array}{l}\text { Res. transfer } \\
\text { cost coefficient }\end{array}$} & \multicolumn{3}{|c|}{$\%$ Improvement in } & \multicolumn{2}{|c|}{ \# Transfers of } \\
\hline & & Total cost & Patient travel cost & \# Patients travelling & Beds & Doctors \\
\hline \multirow{3}{*}{$\mathrm{MoH}$} & 0 & 71.94 & 71.94 & 63.34 & 1141 & 584 \\
\hline & 1 & 70.89 & 71.91 & 63.29 & 86 & 81 \\
\hline & 10 & 62.30 & 71.38 & 61.30 & 88 & 73 \\
\hline \multirow{3}{*}{ SSK } & 0 & 58.78 & 58.78 & 37.47 & 232 & 204 \\
\hline & 1 & 57.83 & 58.80 & 37.47 & 116 & 53 \\
\hline & 10 & 52.16 & 58.68 & 37.43 & 94 & 35 \\
\hline \multirow{3}{*}{ Merged } & 0 & 80.11 & 80.11 & 67.01 & 1769 & 930 \\
\hline & 1 & 78.41 & 80.11 & 67.00 & 39 & 51 \\
\hline & 10 & 63.23 & 78.94 & 65.61 & 42 & 47 \\
\hline
\end{tabular}


transfers, the $\mathrm{MoH}$ and merged networks have feasible assignments. Feasible assignments could not be found even after resource transfers when $\rho_{s i}=0.9$ for $s \in S$.

\subsection{Effect of merging without resource transfers}

Table 8 summarizes the comparisons of optimal costs before and after the merger, without resource transfers. Even if beds and doctors are not re-allocated, the merger results in a better patient-hospital assignment and can achieve a cost reduction of up to $78 \%$. With the re-assignment, both number of patients travelling to another city to get service, and average distance a patient travels is decreased. The benefits of merging decrease slightly with the increasing quality standard, $m_{1 s}$.

\subsection{Interaction of merging and resource transfer}

In previous sections, merging and resource transfer effects were presented separately. Here, we look at the interaction of these two effects. Table 9 shows that there is a synergy between merging and resource transfer. There is more benefit in merging two networks if transferring resources is possible. Similarly, there is more benefit from resources transfers if two networks are merged. Therefore, resource transfers can be used to improve efficiency when networks merge. In this example, the overall improvement is a reduction in cost from 13745750 to 646889 ; this amounts to a decrease of $95.29 \%$. As indicated by the figures for the isolated effects of resource transfers $(65.56 \%)$ and merging $(78.10 \%)$, merging two networks provides more benefits than transferring resources within the two separate networks.

\section{Conclusion}

This paper introduced a model for re-planning hospitals after a merger of hospital networks. The objective is to minimize the patient transportation cost and the cost of changes in

Table 8 Effect of merging without resource transfer

\begin{tabular}{lcc}
\hline$m_{1 s}$ & \multicolumn{2}{c}{$\%$ Improvement in } \\
\cline { 2 - 3 } & Patient travel cost & \# Patients travelling \\
\hline 100 & 78.10 & 67.52 \\
200 & 78.02 & 67.52 \\
400 & 75.11 & 64.67 \\
\hline
\end{tabular}

the system by optimizing resource allocations, service portfolios and assignment of patients to hospitals. The model is based on an assignment formulation, with the addition of resource-transfer decisions which allows for a re-design of the network after the merger. It provides a novel approach to M\&As, with its attention to the resource level of planning after a merger. It also takes into account constraints related with quality standards and hospital closures.

We investigated the potential for cost reduction in this model by applying it to a subset of hospitals in the Turkish hospital system. We had meetings with the head of treatment services department at the Ministry of Health and several specialists and verified our model assumptions with them. Our model serves as a benchmark which shows the potential gains of working on resource allocations to the health authorities.

Our study showed that there was a significant cost reduction opportunity by re-allocating beds and doctors and re-assigning patients after a merger. We found that merging two networks brings significant reduction in transportation costs by a new matching of demand and supply. In addition to the assignment benefits, there was a benefit from re-designing the network by transferring resources between hospitals. The benefits from resource transfer and better assignment of demand and supply are complementary.

Numerical results showed that cost improvements of up to $78 \%$ could be achieved by re-allocation of resources. This surprisingly high improvement result can be attributable to two factors: First, the existing allocation seems to be very inefficient. A comparison of number of resources per 100000 people in each city (Table 3 ) illustrates the inequality in available capacity of different cities. Second, there are some cost components not included in the objective function such as costs of merging and costs of operating the hospitals. Therefore, these results should only be considered as an upper bound on the improvements that could be achieved if resource re-allocation was to be considered in reality.

The model has applications beyond M\&As. It can be useful whenever there is a need, or an opportunity to re-design an existing hospital network. The introduction of new standards for hospitals is an example. If the size of small hospitals will be increased by re-distributing the existing resources in the network, the model can be used with a corresponding constraint on quality standard. The model can also be useful when a hospital is closed and the resources of that hospital

Table 9 Interaction of resource transfer and merging effects on the cost reduction $\left(m_{1 s}=100\right.$ for all $s \in S$ and $\left.m_{2}=1\right)$

\begin{tabular}{lccc}
\hline & \multicolumn{2}{c}{ Total cost } & Reduction by resource transfer \\
\cline { 2 - 4 } & No resource transfer & Resource transfer & $65.56 \%$ \\
\hline No merging & 13745750 & 4733679 & $78.51 \%$ \\
Merging & 3010230 & 646889 & $86.33 \%$ \\
Reduction by merging & $78.10 \%$ & & \\
\hline
\end{tabular}


must be distributed to the remaining ones in the network. Similarly, when a hospital is added to an existing network, the network performance can be enhanced by making changes in the network, taking a holistic perspective.

The model can also be seen as an assessment tool to understand the potential for improving the whole network by a change like merging with another network, introducing a new hospital, or simply increasing the capacity of one hospital.

Finally, although the model presented in this paper is tailored to hospital network problems, it can be modified for use in production-inventory management systems where re-designing facilities can be considered after mergers.

\section{References}

Bretthauer K and Cote M (1998). A model for planning resource requirements in health care organizations. Decis Sci 29(1): 243.

Chu S and Chu L (2000). A modeling framework for hospital location and service allocation. Int Trans Opl Res 7: 539-569.

Clement J, McCue M, Luke R, Bramble J, Rossiter L, Ozcan Y and Pai C-W (1997). Strategic hospital alliances: Impact on financial performance. Health Aff 16: 193-203.

Connor R, Feldman R, Dowd B and Radcliff T (1997). Which types of hospital mergers save consumers money? Health Aff 16(6): $62-74$.

Dokmeci V (1977). A quantitative model to plan regional health facility systems. Mngt Sci 24: 411-419.

The Economist (1999). How to make mergers work. January 9, pp. 15-16.

Galvao R, Espejo L and Boffey B (2002). A hierarchical model for the location of perinatal facilities in the municipality of Rio de Janerio. Eur J Opl Res 138: 495-517.

Geller N and Yochmowitz M (1975). Regional planning of maternity services. Health Serv Res 10: 63-75.

Güneş E and Yaman H (2005). Modeling change in a health system: Implications on patient flows and resource allocations. Clin Invest Med 28: 331-333.

Gupta D and Gerchak Y (2002). Quantifying operational synergies in a merger and acquisiton. Mngt Sci 48: 517-533.
Harper P, Shahani A, Gallagher J and Bowie C (2005). Planning health services with explicit geographical considerations: A stochastic location-allocation approach. Omega 33: 141-152.

Harris J, Ozgen H and Ozcan Y (2000). Do mergers enhance the performance of hospital efficiency? J Opl Res Soc 51: 801-811.

Iyer A and Jain A (2004). Modeling the impact of merging capacity in production-inventory systems. Mngt Sci 50: 1082-1094.

Japsen B (1996). Another record year for dealmaking. Modern Healthcare 26(52): 37-38, 40-41, 44-46.

Morlock L, Waters H, Lyles A, Ozsari S and Aktulay G (2004). A healthy future: Solution suggestions applicable in the health reform. TUSIAD Report, Ankara.

Rahman S and Smith D (1999). Deployment of rural health facilities in a developing country. J Opl Res Soc 50: 892-902.

Rahman S and Smith D (2000). Use of location-allocation models in health service development planning in developing nations. Eur $J$ Opl Res 123: 437-452.

Ruth R (1981). A mixed integer programming model for regional planning of a hospital inpatient service. Mngt Sci 27: 521-533.

Sherman D and Rupert T (2005). Do bank mergers have hidden or foregone value? Realized and unrealized operating synergies in one bank merger. Eur J Opl Res 168: 253-268.

SSK (2007). Statistics Yearbook for the SSK insured population. SSK website, http://www.ssk.gov.tr, accessed 14 January 2008.

Statistics Yearbook (2004). Statistics Yearbook for the Inpatient Treatment Facilities. Turkish Republic Ministry of Health Website, http://www.saglik.gov.tr/istatistikler/ytkiy2004, accessed 5 Jan 2009

Stummer C, Doerner K, Focke A and Heidenberger K (2004). Determining location and size of medical departments in a hospital network: A multiobjective decision support approach. Health Care Mngt Sci 7: 63-71.

Verter V and Lapierre S (2002). Location of preventive health care facilities. Ann Opns Res 110: 121-130.

Weil T (2000). How to enhance the efficacy of health network growth. Int J Health Plann Mngt 15: 17-38.

World Bank (2003). Reforming the health sector for improved access and efficiency. Report no. 24358-tu.

World Bank (2004). Project information document-appraisal stage. Report no. Ab711.

Received February 2008; accepted November 2008 after one revision 